\title{
Service-Differentiated Routing Algorithm in DTN Based on Node Performance
}

\author{
Li Lin-lin, Xia Bing, and He He
}

\begin{abstract}
Considering the characteristics of intermittent interruption and long delay of the Delay/Disruption Tolerant Network (DTN), to improve the message transmission efficiency, a service distinguished routing algorithm based on node performance (NPSR) was proposed. The NPSR algorithm calculates the delivery probability between nodes based on the encounter record. The concept of reference delivery probability is introduced to select relay nodes in priority order. When forwarding copies, the number of copies is determined according to the activity value of nodes. The algorithm is different from traditional method in probability prediction, with differentiated service introduced, and it can control message flow by copies quota. In this way, the efficiency of network is improved and the transmission delay is reduced. The simulation results show that NPSR algorithm has the better performance of message delivery than other routing algorithms.
\end{abstract}

Index Terms-Delay/disruption tolerant network (DTN), routing, node performance, delivery probability, differentiated service.

\section{INTRODUCTION}

Delay/disruption tolerant network (DTN) [1] is a new type of network which used in special application environment. The characteristic of the network is that the node is often intermittent on-off due to various factors [2]. Therefore, the traditional routing algorithm is no longer applicable; the research of the DTN routing algorithm is becoming a hot issue in recent years [3].

There are many classic DTN routing algorithms, including infectious disease algorithm (Epidemic) [4], PROPEHT algorithms [5], Spray and Wait algorithms [6] and so on. Epidemic algorithm [4] is a flooding algorithm based on multiple copy routing mechanism, which increased the connectivity between nodes using the node mobility, exchanged the message, transferred the message which produced by the source node to destination node eventually, and increased the transmission rate by increasing the number of copies. This algorithm is similar to the spread of infectious diseases, which transmit the copy of the messages to all the nodes, in order to improve the probability of successful delivery. The advantages are that it's not need to forecast and estimate the link-state, and its implementation is easily. The disadvantages are that a large number of redundant copies to the network make both the resource utilization and the overall operation efficiency is low. In order to reduce flooding, PROPHET algorithm based on the encounter

Manuscript received October 15, 2016; revised January 12, 2017.

Li Lin-lin, Xia Bing, and He He are with Institute of High and New Technology, Xi’an, China (e-mail: 578709716@qq.com, xbing578@163.com, germanylahm@sina.com). probability between the nodes as the forwarding probability calculation, delivered the message to the nodes which had larger predictive value, so that improved the delivery rate of the message. But the indiscriminate treatment of the messages generated many redundant copies and increased the net work load. The Spray and Wait algorithm from controlled the copy of the message effectively using the quota method. It is divided into 2 stages, the Spray and Wait. In fact, the Spray stage is a kind of flooding, it based on a finite number of neighbors. The source node copied the message for $\mathrm{L}$ samples, and transmitted them to L nodes which they met with independently. If they found the destination node, the message transmission would be end, or they would enter into the Wait stage. The nodes carrying forward copies would be no longer transmitted, instead of waited to meet with destination node and transmitted the message to the destination node. This algorithm can control the network spending effectively, but it can also make the delivery success rate declined at the same time.

In recent years, there are many improved algorithms have been proposed. Reference [7] improved the network utilization and reduced the time delay according to the priority of message unit management on the basis of the Epidemic algorithm. Reference [8] proposed a DTN routing based on delivery probabilistic forecasting. The probability calculation method is different from the PROPHET, and it estimated the delivery probability more accurately based on the historical information among the nodes. Reference [9] proposed the improvement mechanism which used ACK mechanism to remove redundant information and improved the utilization rate using token transmit technology to solve the problems that large cache consume and low efficiency based on the analysis of injection for routing. Reference [10]-[12] made full consideration for the characteristics of node, such as motility, centricity and similarity, which made the calculation result has more abundant connotation. Some new ideas were introduced to routing algorithms, such as [13] introduced Kalman filtering theory, [14] introduced the principle of ant colony algorithm, and these algorithms had improved the performance of the routing to different degrees.

This study proposed a service-differentiated routing algorithm in DTN based on node performance -NPSR algorithm, and it was obtained from the distinction between service and a copy of the quota. This algorithm predicted the delivery probability of the node to the other nodes according to the history of the node information. Then it processed differentiated services according to the different priority of messages. Finally, it distributed the number of transmitting copies equitably according to the active degrees of nodes. It would be more targeted to change the network topology on the basis of making full use of the rule of on and off between 
nodes due to considering the encounter probability difference between different nodes. Besides, distinguishing different priorities for services during message forwarding had enhanced the efficiency of forward and reduced the time delay. In addition, distributing the copies according to the activeness of nodes had reduced the redundant copies and improved the network utilization.

\section{SERVICE-Differentiated Routing Algorithm BASED ON NODE PERFORMANCE}

This study proposed NPSR routing algorithm which main includes delivery probability estimation, differentiated services and copies distribution. Delivery probability estimation counted transmits probability according to the history meeting message of nodes. Differentiated services counted reference probability according to the priority of messages, and then chose the relay node. Copies distribution confirmed the quota of copies according to the performance of nodes, so as to complete the transmitting of copies.

The mathematical symbols of this study are as shown in Table I.

TABLE I: SYMBOL DESCRIPTION

\begin{tabular}{cl}
\hline \hline Symbol & \multicolumn{1}{c}{ The meaning of the symbols } \\
\hline$N$ & The meeting record matrix of this node and other nodes \\
$c(i, k)$ & The collection of all the nodes in the networks \\
$p_{s}(i)$ & The meeting probability of this node $s$ and node $i$ \\
$p_{s}(i, j)$ & $\begin{array}{l}\text { The relay delivery probability from node } s \text { to node } j \\
\text { through node } i\end{array}$ \\
$p_{d, s}(j)$ & $\begin{array}{l}\text { The equivalent delivery probability from node } s \text { to node } \\
C\end{array}$ \\
$p_{r, s}(j)$ & The parameter of messages priority \\
& $j$
\end{tabular}

\section{A. The Probabilistic Forecasting Based on Meeting Messages' History}

Fig. 1 showed the simple diagram of the DTN network, the dashed line means the connection between nodes is intermittent interrupted connection (intermittent meeting), the location of the nodes in the diagram doesn't mean the actual relative position.

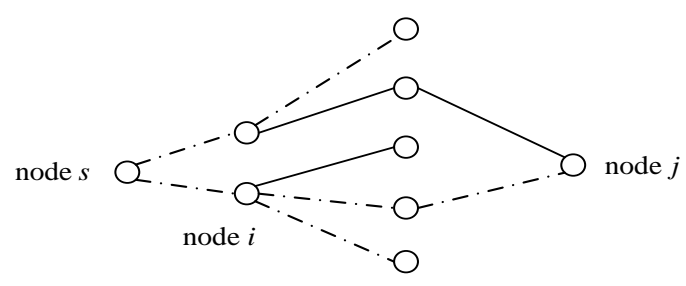

Fig. 1. The simple diagram of the network.

The NPSR routing algorithm used the probability computing method based on history information, and every node maintains a meeting record matrix $C M$.

Definition 1 Meeting record matrix $C M$.

$$
C M=\{c(i, k)|1 \leq i \leq| N \mid, 1 \leq k \leq K\}
$$

$C M$ recorded the meeting situation of the current node with the other nodes in a recent period of time (take $K$ time units). If the current node is $s$, and node $s$ connected with node $s$ at $k$ th $(1 \leq k \leq K)$ time unit, then the corresponding element $c(i, k)=1$ in the matrix, or $c(i, k)=0$.Every row of the matrix is a queue which length is $K$ essentially, the queue would move forward a step when status updates every time, and the state at $K$ the time unit would join the tail of the queue. In this way, the meeting information of the node in recent period of time would ensure the record of the meeting record matrix.

The meeting probability of the current node $\mathrm{s}$ with the other nodes could be received according to the meeting record matrix. The meeting probability of the node $s$ with node $i$ at lately $K$ time units could be used to express the meeting probability $p_{s}(i)$ of two nodes at next time unit.

$$
p_{s}(i)=\frac{\sum c(i, k)}{K}
$$

Obviously, the more frequently the nodes meeting in a recent period of time, the more likely to be meet in the next time, or it would decrease. The meeting records and the meeting probability would update at the end of each time unit.

Assumes that the destination node is $j$, in order to count the relay delivery probability that the message of node $s$ send to node $j$ through node $i p_{s}(i, j)$, the equivalent delivery probability from node $i$ to node $j p_{d, i}(j)$ should be counted first. It is should be explanted that the equivalent delivery probability of two nodes is usually not only one hop or one link. In fact, it is multiple paths come down to a general delivery probability between two nodes.

There are two possible paths from node $i$ to node $j$, that through or not through node $s$.

$$
p_{d, i}(j)=1-\left(1-p_{i}(s, j)\right)\left(1-p_{d, i, s-}(j)\right)
$$

In this equation, $p_{d, i, s-}(j)$ means the general delivery probability that from node $i$ to node $j$ not through node $s$, it can be received that

$$
p_{d, i, s-}(j)=1-\frac{1-p_{d, i}(j)}{1-p_{i}(s, j)}
$$

So, the relay delivery probability that the message of node $s$ send to node $j$ through node $i \quad p_{s}(i, j)$ could be expressed that

$$
p_{s}(i, j)=p_{s}(i) \times p_{d, i, s-}(j)
$$

The equivalent delivery probability from node $s$ to node $j$ could be count from the relay delivery probability

$$
p_{d, s}(j)=1-\prod_{i=1}^{N}\left(1-p_{s}(i, j)\right)
$$


When node $s$ meet with node $i$, the relay delivery probability $p_{s}(i, j)$ and the equivalent delivery probability from node $s$ to node $j p_{d, s}(j)$ could be received according to (4) and (5). The pseudo-code of the delivery probability algorithm is shown in Algorithm $\mathrm{I}$.

ALGORITHM I: THE DELIVERY PROBABILITY

INPUT: current_time
1. IF ( current_time\%time_unit $=0$ ) THEN
2. UPDATE $C M$
3. UPDATE $p_{S}(i)$ as $(1)$ for each node $i$
4. IF node $s$ contact node $i$ THEN
5. UPDATE $p_{S}(i, j)$ and $p_{d, s}(j)$ as $(4)(5)$ for each node $j$
6. END IF
7. END IF

\section{B. The Differentiated Service Based on the Message Priority}

The differentiated service means distinguish different priority of messages to service. It should be increased its forwarding probability when a high-priority message being forwarded, so that the messages' copy quantity could be kept at a higher level and it could also improve the accessibility. The second-rate messages should be forwarded in a normal probability. The balanced network traffic should be taken as the principal thing when forwarded the low priority messages And it could make less copy of the message transmission in the network, the accessibility was also correspondingly reduced.

According to the different priorities, this study adopted the reference probability. The relationship between the reference probability and the delivery probability is

$$
p_{r, s}(j)=C \cdot p_{d, s}(j)
$$

When the priority is high, $C<1$. When the priority is middle, $C=1$. When the priority is low, $C>1$. Thus it can be seen that the messages with different priorities still have different reference delivery probability under the same meeting probability.

With two jump retransmission as an example to analyze the use of the reference delivery probability, assumption for the current node is $s$ carrying message $m$, and its destination node is $j$. When node $s$ meet with node $i$, compared the relay delivery probability $p_{s}(i, j)$ of node $i$ with the reference delivery probability $p_{r, s}(j)$ of node $s$, if $p_{s}(i, j)>p_{r, s}(j)$, then transmit this message, or don't transmit it. It is easy to get this result that $p_{r, s}(j)>p_{d, s}(j)$ when the priority of the message is low. There are $l_{1}$ meeting nodes of node $s$ whose relay delivery probability are greater than their equivalent delivery probability $p_{d, s}(j)$, and there are $l_{2}$ nodes whose relay probability are greater than the reference delivery probability $p_{r, s}(j)$. If it didn't introduced the reference probability to conduct the differentiated service, there would be $l_{1}$ relay nodes would receive the retransmission copies. And if it considered the reference probability, there would be $l_{2}$ relay nodes could receive the retransmission copies. Because $p_{r, s}(j)>p_{d, s}(j)$, the number of relay nodes under the condition of introduced the reference probability is $l_{2}$ which is less than that haven't introduced the reference probability which is $l_{1}$. Obviously, the differentiated service limited the forwarding of low priority messages through reducing the number of the relay node. But the forwarding of high priority messages is on the contrary.

Therefore, the reference delivery probability of the high priority messages decreased in corresponding, and there would be more relay nodes satisfied with the requirement of retransmission copies, thus it would improve the transmission efficiency. The reference delivery probability of the middle priority messages was equal to the equivalent delivery probability, and it would be forwarded according to the normal process. The reference delivery probability of the low priority messages increased in corresponding, and the number of relay nodes which could forward messages would be limited so that the transmission efficiency would be reduced.

\section{The Allocation Strategy of Copies Based on the Activity of Nodes}

It is necessary to focus on solving the problem of that the allocation strategy of retransmission copies after decided the relay node, namely how many message copies would be distributed to the relay node. The current node connected and exchanged information with relay node, but it didn't mean that the copies of the current node had transmitted to the relay node completely. This was because of that the capacity of the current node was equal to the relay node. The current node should retain some copies or it would lead to invalid transfer for a large number of copies.

The activity of nodes was as an important indicator to evaluate the performance of nodes, the higher activity of nodes would have a greater chance to connect with the other nodes, and the transmission efficiency would be higher if the message copies transmitted to this kind of node. It is generally believed that the node contacted more nodes the higher activity it had. Therefore, the number of meeting nodes showed the activity of node. Each node maintains a meeting node vector quantity called $E$.

Definition 2 The gather vector quantity of the meeting node called $E$.

$$
E=\{i|1 \leq i \leq| N|, 0 \leq| E|\leq| N \mid\}
$$

$E$ records the collection that the current node meeting with every node. Firstly, checking whether the node $i$ was included in the collection $E$ when node $s$ connected with node $i$. If it didn't in the collection $E$, put node $i$ into the collection $E$. The value of $|E|$ was the activity of node, and its initial value was 0 and no more than $|N|$.

One kind of allocation strategy was distributed according to the proportion of activity. Node $s$ carrying $L$ copies of message $m$, its destination node was $j$, the relay nodes 
which conformed to the conditions were $i$ respectively, then the relay node would be assigned $L_{i}$ copies in corresponding. According to the analysis above, the higher activity of node was, the more message copies would be distributed. The lower activity of node was, the less message copies would be distributed. According to this principle, the computing method of counting the number of copies that the relay node $i$ should be got was as shown in (7).

$$
L_{i}=\frac{\left|E_{i}\right|}{\left|E_{i}\right|+\left|E_{s}\right|} \cdot L
$$

Among them, $\left|E_{s}\right|$ means the activity of node $s,\left|E_{i}\right|$ means the activity of node $i$. At this moment, the rest number of copies for node $s$ was

$$
L_{s}=\frac{\left|E_{s}\right|}{\left|E_{i}\right|+\left|E_{s}\right|} \cdot L
$$

The pseudo-code of copies forwarding algorithm is shown in Algorithm II.

ALGORITHM II: COPIES FORWARDING
\begin{tabular}{|l}
\hline INPUT: node $s$ met with node $i$ \\
1. FOR each message $m$ in buffer of $s$ \\
2. $j \leftarrow$ destination node of $m$ \\
3. UPDATE $p_{d, s}(j)$ and $p_{s}(i, j)$ \\
4. $C \leftarrow \operatorname{priority}$ of $m$ \\
5. UPDATE $p_{r, s}(j)$ \\
6. IF $\left(p_{s}(i, j)>p_{r, s}(j)\right)$ THEN \\
7. INSERT $m$ into delivery list \\
8. $L_{i} \leftarrow \frac{\left|E_{i}\right|}{\left|E_{i}\right|+\left|E_{S}\right|} \cdot L$ \\
9. $L_{S} \leftarrow \frac{\left|E_{s}\right|}{\left|E_{i}\right|+\left|E_{s}\right|} \cdot L$ \\
10. IF $L_{i}=0$ \\
11. DELETE $m$ from delivery list \\
12. END IF \\
13. SEND replicas of $m$ to node $i$ \\
14. IF $L_{s}=0$ \\
15. DELETE $m$ from buffer of $s$ \\
16. END IF \\
17. END FOR
\end{tabular}

\section{PERFORMANCE ANALYSIS}

\section{A. Simulation Environment Setting}

TABLE II: SimULATION PARAMETER SETTING

\begin{tabular}{cc}
\hline \hline Simulation parameter & Value \\
\hline Area & $2000 \mathrm{~m} \times 2000 \mathrm{~m}$ \\
Number of nodes & 30 \\
Message size & $250 \mathrm{~KB}$ \\
Interval that messages generated & $30 \mathrm{~s}$ \\
Sending rate of node data & $128 \mathrm{~KB} / \mathrm{s}$ \\
Buffer memory space of node & $2-10 \mathrm{MB}$ \\
Survival time & $120 \mathrm{~min}$ \\
Simulation time & $20000 \mathrm{~s}$ \\
\hline
\end{tabular}

This study conducted simulation experiment to analyze and compare the performance of NPSR with two popular routing algorithms, Epidemic and PROPHET. All simulations were performed in the simulator The ONE [15]. The area of the simulation scene was $2000 \mathrm{~m} \times 2000 \mathrm{~m}$, the number of nodes was 30, 5 of them were fixed nodes and 25 of them were mobile nodes. The simulation time was 20000s. The simulation experiment evaluated the routing algorithm through 2 performance indicators. It analyzed based on the change of the buffer memory space of node and message size. The simulation parameters were shown in Table II.

\section{B. The Analysis of Simulation Result}

1) The performance comparison under the change of the buffer memory space.

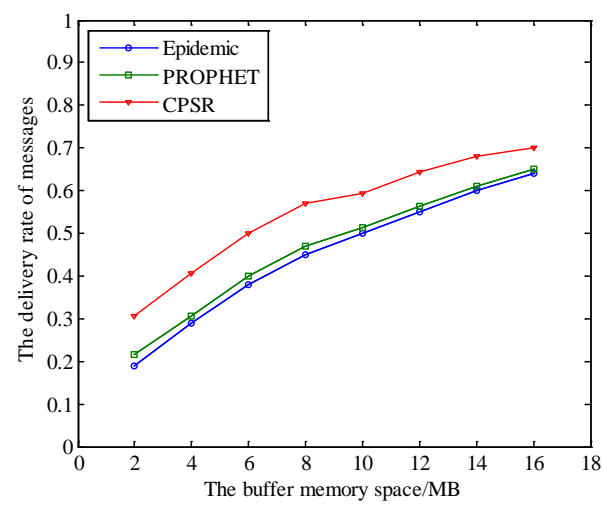

(a) The delivery rate of messages

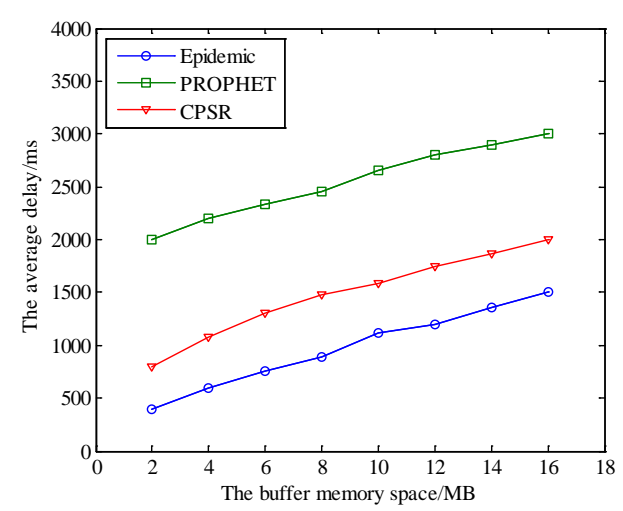

(b) The average delay

Fig. 2. The size of the node buffer memory space effects on algorithm performance.

Fig. 2(a) described the buffer memory space of node influenced the message delivery rate. It could be seen from the figure that the message delivery rate of NPSR was superior to Epidemic and PROPHET. With the increase of the buffer memory space of node, the message delivery rate of 3 algorithms were all increasing. It was because that the buffer memory space increasing allowed the node carrying more messages, and more messages could be forwarded effectively, the message delivery rate was also improved. In the Epidemic algorithm, the node copied and forwarded the messages to all the nodes that it met directly. This approach didn't consider the rule of nodes connection, it also had no targeted control to the flow direction of messages, and its delivery success rate was low relatively. The PROPHET algorithm had considered the rule of node meeting, and it forwarded the message to the nodes which could meet destination node easier, so its delivery success rate was higher than the Epidemic algorithm. The adaptation on 
forwarding probability updating of the NPSR algorithm was better. This algorithm distinguished the messages reasonably according to the different priorities, guided the messages forwarding to the destination node which could be reached easier. It balanced the network load and improved the efficiency of delivery so that it had higher delivery success rate than the PROPHET algorithm.

Fig. 2 (b) described the size of the node buffer memory space effects on the average delay. It could be seen from the figure that the average delay increased as the buffer memory space increasing. It was because that the increasing of the buffer memory space must lead to the node carrying more messages, and the message could not be forwarded in time leaded to it was carried in the cache for a long time, so that the delay increased. Among them, the average delay of the NPSR was lower than the PROPHET but higher than the Epidemic. It was because that the Epidemic algorithm used flooding mechanism. And it did not need to forecast the link-state estimate, either needs node selection. Its implementation process was simple so that the average delay was smaller. The PROPHET and NPSR algorithm were all considered the forecast the link-state estimate and node selection, which required a lot of time to do the information and relevant calculation. The latter distinguished the messages reasonably according to the different priorities when it was forwarding the messages, which had reduced unnecessary forwarding process so that it had shorter delay than the prophet.

2) The comparison of the performance under the change of the message size

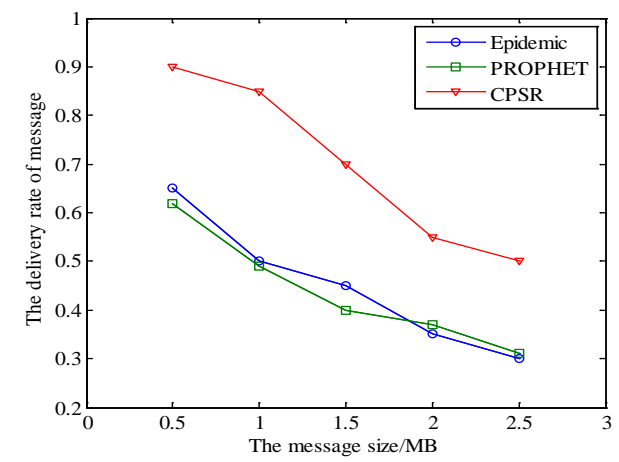

(a) The delivery rate of message.

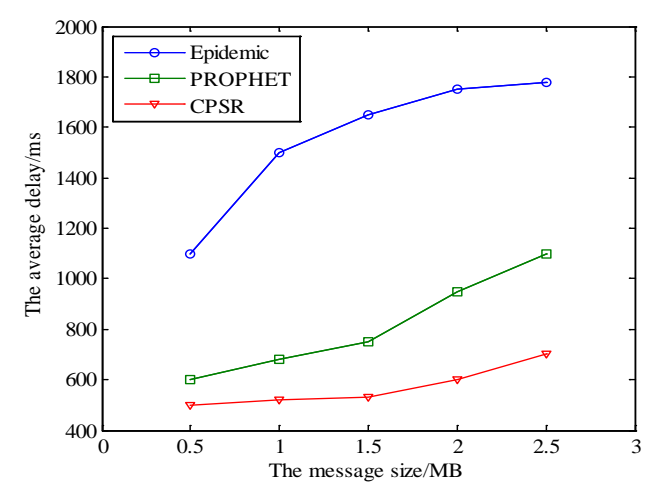

(b) The average delay.

Fig. 3. The message size effect on the algorithm performance.

Fig. 3(a) described the change of the message size effected the message delivery rate. It was can be seen from the figure that the delivery rate of the 3 algorithms was declined with the increasing of the message size. It was because that the increasing of the message size could lead to reduce the number of messages that the node carrying under the restriction of the buffer memory space, and some messages couldn't be kept for a long time but failed to be forwarded. The Epidemic algorithm used flooding mechanism, namely meeting and forwarded. It would be soon to reach the limitation of network load with the increasing of the message size so the delivery rate was lower than the PROPHET and NPSR. The NPSR algorithm introduced the concept of reference probability when forwarding the message. It selected forwarding nodes which were more suitable for the different service level messages, and it also controlled the number of copies more accurate when forwarding, so the NPSR algorithm was still having higher message delivery rate than the PROPHET algorithm even under the increasing of the message size situation.

Fig. 3(b) described the change of the message size effected the average delay. It was can be seen from the figure that the average delay of the 3 algorithm was increasing as the increasing of the message size. It was because that the increasing of the message size would take up a lot of buffer memory space so that made the message congestion, which leaded to the message couldn't be forwarded in time and increased the average time delay. Among them, the time delay of the Epidemic algorithm was greater than the other two algorithms. It was because that the messages spread quickly using a similar virus message transmission mechanism. And at the same time it was also generated a large amount of redundancy easily which made the network utilization reach to saturation and affected the efficiency of the message forwarding seriously, so that the average delay was the largest. While the NPSR and PROPHET algorithm were all adopt the probabilistic routing method. Every time the copies were delivered to the optimal relay node, but the NPSR algorithm had made an equitable distribution for the number of the copies according to the activity of the nodes, controlled the copy redundancy effectively, so that its average time delay was smaller.

\section{CONCLUSION}

This study had done several aspects as followings for the DTN network routing problem.

It had proposed the NPSR routing algorithm for DTN network. This algorithm proceeded the counting of the forwarding probabilistic forecasting and update between nodes. And this algorithm was even more conformed to the rule of the node movement.

The NPSR routing algorithm had introduced the idea of the differentiated service. It distinguished the messages in the convection communication according to the different brackets, which could improve the transmission efficiency of message.

It had made an equitable distribution for the number of the copies according to the activity of the nodes and improved the network utilization.

Compared the routing performance of the NPSR algorithm with the Epidemic and prophet algorithm, the simulation result showed that the routing performance of the NPSR 
algorithm was better than the Epidemic and prophet algorithm in the meteor trail communication system.

The next step will apply the conclusion of this study to the more complex meteor trail communication system, and put forward more efficient routing algorithm.

\section{REFERENCES}

[1] J. S. Su, Q. l. Hu, B. k. Zhao, and W. Peng, "Routing techniques on delay/disruption tolerant networks,” Journal of Software, vol. 21, pp. 119-132, Jan. 2010.

[2] C. Lin, Y. W. Dong, and Z. G. Shan., "Research on space internetworking service based on DTN," Journal of Computer Research and Development, vol. 51, pp. 931-943, May 2014.

[3] Z. Zhang, "Routing in intermittently connected mobile ad hoc networks and delay tolerant networks: Overview and challenges," IEEE Communications Surveys and Tutorials, vol. 8, pp. 24, Jan. 2006.

[4] A. Vahdat and D. Becker, "Epidemic routing for partially-connected ad hoc networks,” Duke University, Durham, USA: Technical Report CS-2000-06, 2000

[5] H. A. Nguyen, S. Giordano, and A. Puiatti, "Probabilistic routing protocol for intermittently connected mobile ad hoc network," In Proc. of IEEE International Symposium on a World of Wireless, pp. 1-6, 2007.

[6] T. Spyropoulos, K. Psounis, and C. S. Raghavendra, "Spray and wait: Efficient routing in intermittently connected mobile networks,” ACM Sigcomm WDTN, pp. 252-259, 2005.

[7] H. Guo, X. W. Wang, M. Huang, and D. D Jiang, “Adaptive epidemic routing algorithm based on multi queue in DTN,” Journal of Chinese Computer System, vol. 33, pp. 829-832, Apr. 2012.

[8] M. Peng, P. L. Hong, K. P. Xue, and H. C Lu, "Delivey probability prediction based efficient routing in DTN,” Journal of Computers, vol. 34, pp. 174-181, Jan. 2011.

[9] E. Zheng and Q. X. Luo, "Spray and wait routing based on ACK-mechanism in disruption tolerant networks," Journal of Computer Applications, vol. 32, pp. 367-369, Feb. 2012.

[10] Z. J. Zhang, Z. G. Jin, and Y. T Shu, "Efficient routing in social DTN based on nodes' movement prediction,” Chinese Journal of Computers, vol. 36, pp. 626-635, Mar. 2013.

[11] Y. H. Wu, S. Deng, and H. B. Huang, "Research of situation-aware routing method in delay tolerant network,” Journal of Electronics \& Information Technology, vol.33, pp. 575-579, Mar. 2011.
[12] X. Wang and R. X He, "An improved probabilistic routing algorithm based on multi-information fusion in delay tolerant networks," Telecommunication Engineering, vol. 54, pp. 1569-1575, Nov. 2014.

[13] Y. B. Chen, "Self-adaptive context aware routing protocol for delay and tolerant network based on Kalman filter theory," Application Research of Computers, vol.32, pp. 838-845, Mar. 2015.

[14] G. H. Deng, W. H. Cao, J. Zhang, L. Feng, and X. Cheng, "Data dissemination mechanism with network coding based on ant colony algorithm in DTN environment,” ACTA Electronica Sinicadtn, vol. 42, pp.1636-1641, Oct. 2014.

[15] A. Keranen, J. Ott, and T. Karkkainen, “The ONE simulator for DTN protocol evaluation," In Proc. of the 2nd International Conference on Simulation Tools and Techniques. Rome, pp. 1-10, 2009.

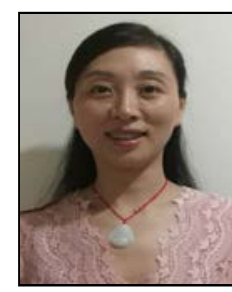

Li Lin-lin received the $\mathrm{PhD}$ degree in computer science and technology, in Institute of High and New Technology, Xi'an, China, in 2005. Her research interests are computer networks and system simulation.

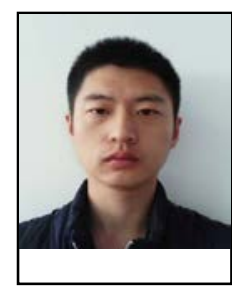

Xia Bing received the MS degree in computer science and technology, in Institute of High and New Technology, Xi'an, China, in 2016. His research interests are computer networks and delay/disruption tolerant network.

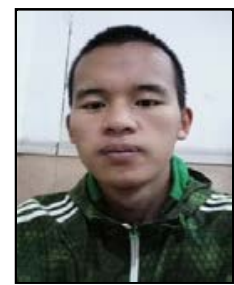

He He is currently working towards a MS degree in computer science and technology, in Institute of High and New Technology, Xi'an, China. His research interest is delay/disruption tolerant network. 